Europe's Journal of Psychology, 7(4), pp. 604-623

www.ejop.org

\title{
Emotion regulation difficulties in alexithymia and mental health
}

\author{
Rakesh Pandey \\ Prachi Saxena \\ Akanksha Dubey \\ Department of Psychology, Banaras Hindu University
}

\begin{abstract}
Alexithymia, characterized by difficulty in identifying and describing feelings and a deficit in the cognitive modulation of emotions, has been linked with health related problems. Indirect empirical evidences suggest the possibility that alexithymia may potentially also relate with emotion regulation difficulties. However, the exact nature of the relationship between the two constructs has not been fully explored. The relationship between the two constructs may reflect the overlap of the content domains or dependence of one (e.g., emotion regulation difficulties) on the other (e.g., alexithymia). Given the link between the two constructs, it is also likely that alexithymics' health related difficulties may result from their impaired ability to regulate emotions. The present study tested the above theoretical propositions on 27 alexithymic and 26 nonalexithymic participants who were assessed on self-report measures of emotion regulation difficulties and general mental health. The findings revealed that compared to non-alexithymics, the alexithymics showed greater emotion regulation difficulties and such affective difficulties differentiated the two groups with a very high accuracy. Further, the observation of two orthogonal components (emotion regulation difficulties and alexithymia) with a minimum overlap suggests that these constructs represent two independent domains of behavior. Findings also revealed that alexithymics reported more health related problems as compared to non-alexithymics and the health problems of alexithymics are likely to be an outcome of their emotion regulation difficulties (indicated by a non-significant health difference after controlling for emotion regulation difficulties). The findings imply that alexithymia is associated with emotion regulation difficulties and such difficulties largely contribute to health related problems.
\end{abstract}

Keywords: alexithymia, emotion regulation difficulties, emotion regulation, mental health. 
Alexithymia is a personality construct characterized by the difficulty in identifying and describing one's own feelings and emotions. It is conceptualized as a multidimensional construct encompassing the following four dimensions: (i) difficulty in identifying and describing feelings, (ii) difficulty in distinguishing between feelings and bodily sensations, (iii) impaired symbolic activity as evidenced by paucity of fantasy and other imaginative activity, and (iv) externally oriented thinking i.e., a preference for focusing on external events rather than inner experiences (Taylor, 1994, 1984).

Alexithymia as a trait is associated with a deficit in cognitive modulation of emotions (Taylor, 1994) and therefore, is likely to be associated with emotion regulation difficulties. Researchers have theorized that alexithymia reflects a deficit in the cognitive processing and regulation of emotions, and that the disorders with which alexithymia is most strongly associated can be conceptualized as "disorders of emotion regulation" (Taylor, 1994; Taylor, Bagby, \& Parker, 1997). However, efforts to empirically validate such theoretical speculations are scant. Moreover, the few empirical studies that aimed at exploring the alexithymia-emotion regulation link have utilized different conceptual/operational definition of emotion regulation. For instance, a study examined the correlations between alexithymia and the recognition and regulation of emotions in a sample of healthy subjects and the findings revealed that objectively assessed emotion recognition correlated negatively with external thinking and positively with the importance of emotional introspection (Kessler, Kammerer, Hoffmann, \& Trave, 2010). Further, this study demonstrated that expressive suppression correlated with difficulty in identifying and describing feelings, a core feature of alexithymia. In a recent study (Connelly \& Denney, 2007) it was noted that those with high levels of the alexithymic trait appeared to manifest chronically elevated subjective negative affect relative to autonomic activity regardless of the level of environmental demands. The empirical observations that alexithymic individuals have greater propensity towards negative emotions and show a tendency to suppress emotional expression indirectly support the possibility that alexithymic individuals may have emotion regulation difficulties. A number of other indirect empirical evidences also support the possibility of emotion regulation difficulties in alexithymia. Several studies have reported that alexithymia is associated positively with maladaptive styles of emotion regulation (such as bingeing on food or drinking alcohol) and negatively with adaptive behaviors, such as thinking about and trying to understand distressing feelings or talking to a caring person (Bekendam 1997; Parker, Taylor, \& Bagby, 1998). Findings demonstrating the association of alexithymia with insecure attachment styles (Schaffer, 1993; Scheidt \& 
Waller, 1999) also provide indirect evidence for the link between alexithymia and less effective emotion regulating skills.

The body of research exploring emotion regulation difficulties in alexithymia, although promising, shows that more work is needed to expand our understanding of the relationships between the two constructs. This becomes a much-needed undertaking in the light of the reported variations in the conceptualization of emotion regulation. Some studies considered reduced emotion recognition capacity as an index of difficulty in emotion regulation (e.g., Kessler et al., 2010) while others considered the tendency to experience negative emotions (in response to one's own emotional reactions - an indicator of a lack of emotional acceptance) as emotion regulation difficulty (Cole, Michel, \& Teti., 1994; Greenberg \& Paivio, 1998; Hayes, Strosahl, \& Wilson, 1999). Researchers have sometimes defined emotion regulation as control of emotional experience and expression (Garner \& Spears, 2001) and in other places as the capacity to experience and differentiate a full range of emotions (Cole et al., 1994; Gross \& Munoz, 1995). Similarly, the ability to accept and value emotional responses has also been considered to be a kind of emotion regulation by some researchers (Cole et al., 1994). These variations in the conceptualization of emotion regulation suggest its multidimensional nature. Further, most of the attempts to explore emotion regulation difficulties focused on one or two components of the multidimensional construct of emotion regulation. Thus, to uncover the nature of the relationship between emotion regulation and alexithymia we need to explore how and to what extent the various components of emotion regulation correlate with various dimensions of alexithymia using a psychometrically sound and comprehensive measure. Keeping this in view, the current study adopts a multidimensional conceptual model of emotion regulation (as proposed by Gratz and Roemer, 2004). According to this model emotion regulation consists of four dimensions: (a) awareness and understanding of emotions, (b) acceptance of emotions, (c) ability to control impulsive behaviours and behave in accordance with desired goals when experiencing negative emotions, (d) ability to use situationally appropriate emotion regulation strategies, flexibly to modulate emotional responses as desired in order to meet individual goals and situational demands.

It is also evident from the preceding review that alexithymia and emotion regulation difficulties may not only potentially relate with each other but some minimal overlap between the constructs is also possible. For instance, it is evident from the above discussion of the conceptual models of alexithymia and emotion regulation that 'awareness and understanding of emotions is an important component of both alexithymia and emotion regulation. Such observation suggests the theoretical possibility of similarity (overlap) in the content of the two constructs. However, apart 
from the abovementioned component, other components of emotion regulation (e.g., ability to control impulsive behaviour, ability to use appropriate emotion regulation strategies) appear to be distinct from alexithymia and thus it is likely that the two constructs might be largely independent and/or represent different constructs. These theoretical propositions, however, must be put to empirical test so that the nature of the relationship between the two constructs is revealed. In other words, there is a need to examine and empirically address such theoretical possibilities as: i) alexithymia is characterized by various types of emotion regulation difficulties but these difficulties themselves are not the defining components of alexithymia, ii) alexithymia is associated with emotion regulation difficulties and some types of emotion regulation difficulties (e.g., lack of awareness and/or understanding of emotions) may in fact represents some component or dimension of alexithymia.

The potential relationship (or overlap) between alexithymia and emotion regulation difficulties is further supported indirectly by the observations that both constructs have shown a similar pattern of association with mental and physical health related problems such as hypertension, cardiovascular disease, depression, sleep disorder (Bavermann, Parker, \& Taylor, 2008; Byrne \& Ditto, 2005; HonKalampi et al., 2010; Jula, Salminen \& Saarijarvi, 1999). For instance, although alexithymia was initially linked with various psychosomatic disorders (Taylor, 1994), latter it was found to be associated with a variety of other types of mental health problems such as depression (Honkalampi et al., 2010), anxiety disorders (Devine, Stewart, \& Watt, 1999), insomnia and sleep related problems (Bavermann, Parker, \& Taylor, 2002; Bavermann et al., 2008). For example, in a study the depressive symptom severity was found to be strongly associated with alexithymia particularly with two of its dimensions - difficulty in identifying feelings and difficulty in describing feelings (Bamonti et al., 2010).

Similarly, higher prevalence of alexithymia in patients with anxiety and related disorders has also been reported. For instance, Devine and colleagues (Devine, et al., 1999) observed that individuals who experience frequent anxiety and greatly fear their anxiety symptoms report the greatest difficulties identifying and describing emotional states (an alexithymic-like difficulty). Similarly, Parker and colleagues (Parker, Taylor, Bagby, \& Acklin, 1993) observed that a majority of patients with panic disorder had alexithymia and alexithymic individuals report less ego strength and significantly higher levels of anxiety and somatic complaints. 
Similarly, there are abundant research evidences that relate emotion regulation difficulties with a variety of health related problems such as depression (Gross \& Munoz, 1995), anxiety disorders (Mennin, McLaughlin, \& Flanagan, 2009), post traumatic stress disorder (McDermott, Tull, Gratz, Daughters, \& Lejuez, 2009), and panic disorder (Tull \& Roemer, 2007) etc. Some researchers (e.g., Campbell-Sills \& Barlow, Brown \& Hofmann 2006), have argued that individuals with anxiety and mood disorders make counterproductive attempts to regulate acute affective episodes that lead to the exacerbation and persistence of unwanted emotion (i.e., ineffective emotion regulation). Similarly, McDermott and colleagues (2009) supported the central role of difficulties in emotion regulation in the prediction of PTSD within a crack/cocaine dependent population. Studies also report that patients with panic attack disorder show reduced emotional clarity and difficulty to distinguish between bodily sensations and emotional states, resulting in internal states being perceived as ambiguous and unpredictable (Tull \& Roemer, 2007). Difficulties in emotion regulation have also been associated with various personality disorders (Gratz, Tull, \& Gunderson, 2008) and binge eating (Whiteside et al., 2007).

From the above review it is evident that alexithymia and emotion regulation difficulties may potentially correlate with each other and it is likely that some of the observed relationship may be because of the similarity (overlap) in the content of both these constructs. However, empirical tests of such speculation have yet to be conducted. Furthermore, the review also indicates that despite the encouraging research evidence that emotion regulation difficulties correlate with alexithymia and both of these constructs are related with health related problems, the potential mediating role of emotion regulation difficulties in explaining the alexithymia - health relationship has not yet been properly explored. Given the relationship of emotion regulation difficulties with both alexithymia and mental health problems, it can be hypothesized that the mental health problems of alexithymics may be caused by their difficulties in regulating emotions. In other words, the alexithymia-health relationship may be considered to be mediated by emotion regulation difficulties. However, again, such theoretical speculations need to be empirically tested.

Taking into consideration the gaps in the evidence, the present study makes an attempt to examine how far alexithymia may be characterized by emotion regulation difficulties and to what extent such emotional difficulties may account for the widely reported health related problems in alexithymics. To explore the role of emotion regulation difficulties in alexithymia, alexithymic and non-alexithymic individuals were compared on a comprehensive measure of emotion regulation difficulties and discriminant function analysis was conducted to explore the level of accuracy with which emotion regulation difficulties can differentiate between them. 
Apart from this, an effort was also made to address the issue of potential overlap betweem the two constructs (alexithymia and emotion regulation difficulties) using the factor analytic approach. To address this issue we conducted a factor analysis on the inter-correlation matrix obtained by combining the dimensions of alexithymia and emotion regulation difficulties. Our assumption is that, if both the constructs are orthogonal (no-overlap), then the analysis should yield two factors: one composed of dimensions of alexithymia and emotion regulation difficulties respectively. Contrary to this, if there is overlap then some dimensions of both constructs will cluster together to form a factor. Further, to test the hypothesis that the mental health problems of alexithymics may be caused by their difficulties in regulating emotions, the differences in mental health problems of alexithymic and nonalexithymic participants were examined and compared before and after controlling the variance attributable to emotion regulation difficulties. The findings of the study thus aim to develop insight into the affective dynamics of health and to uncover the nature of the relationship between alexithymia and emotion regulation difficulties.

\section{Method}

Participants

Initially 104 students (64 males and 40 females) in the age range of 18 to 27 years (mean age $=21.19, \mathrm{SD}=2.51$ years) were assessed on a measure of alexithymia and using quartile split the $25 \%$ low scorers (non-alexithymic) and the $25 \%$ high scorers (alexithymic) were finally selected for the present study. In the alexithymic group there were 27 participants (16 male \& 11 female) whereas 26 participants (16 male \& 10 female) were placed in the non-alexithymic group. The mean alexithymia score of alexithymic group was 62.70 (SD =3.58) and that of non-alexithymic group was 35.12 (SD = 5.89). The two groups differed significantly in terms of alexithymia scores $(t(51)=20.68, p<.001)$. The age range of alexithymic participants was 18 to 26 years (mean age $=21.15$ years, $S D=2.16$ years) and that of non-alexithymics was 18 to 26 years (mean age $=22$ years, SD $=2.71$ years). None of the participants reported any present or prior history of medical or psychiatric illness in an interview conducted before the administration of the tools for the present study.

\section{Measures}

The Hindi version of 20 item Toronto Alexithymia Scale (TAS-20-H; Pandey, Mandal, Taylor, \& Parker, 1996) was used to assess the level of alexithymia of the participants. The TAS-20-H assesses three dimensions of alexithymia: (a) difficulty in identifying 
feelings and distinguishing them from bodily sensations, (b) difficulty in describing feelings to others, and (c) externally oriented thinking. Respondents use a 5-point Likert scale (from (1) 'strongly disagree' to (5) 'strongly agree') to rate themselves on statements describing alexithymic traits. The negatively keyed items are scored in reverse order. Higher score on this scale indicates higher levels of alexithymia.

Emotion regulation difficulties were assessed using a Hindi adaptation of the Difficulties in Emotion Regulation Scale (DERS; Gratz \& Roemer, 2004). The DERS is a 36 item measure that assesses difficulties in regulating emotions across six domains: (a) non-acceptance of negative emotions; (b) difficulties in engaging in goaldirected behaviours when experiencing negative emotions; (c) difficulties in controlling impulsive behaviour; (d) lack of emotional awareness; (e) limited access to emotion regulation strategies; (f) lack of emotional clarity. Participants were asked to indicate how often the items apply to themselves, with responses ranging from 1 to 5 , where 1 is 'almost never' (0-10\%); 2 is 'sometimes' (11-35\%); 3 is 'about half the time' (36-65\%); 4 is 'most of the time' (66-90\%); and 5 is 'almost always' (91-100\%). The negatively keyed items are reverse scored and thus, the higher score indicates greater difficulties in regulating emotions.

The Hindi version of the DERS was developed using the typical method of translation and back-translation. The initial psychometric evaluation of the Hindi version of DERS (the DERS-H) revealed that the scale is psychometrically sound and reliable (Pandey, Saxena, Gupta, \& Dubey, 2010). The reliability of the DERS-H was found to be .90 and most of the subscales demonstrated satisfactory internal consistency (alpha $=.80$ or higher). One subscale (lack of emotional awareness), however, showed a relatively lower level of reliability (alpha $=.606$ ).

The 28 item version of the General Health Questionnaire (GHQ-28; Goldberg \& Hillier, 1979) was used to assess four dimensions of mental health, namely somatic complains, anxiety \& insomnia, depression and social dysfunction. Each item in this questionnaire was followed by four response alternatives with score credit ranging from 1 to 4. The response alternatives have been arranged in such a way that higher score on the scale indicate better health. For the present study, a Hindi version of the GHQ-28 was developed following the cross-cultural methodology (translation - back translation) of establishing transliteral equivalence.

Procedure

The participants of the present study were contacted either individually or in small groups consisting of 3 to 4 persons. Before the administration of the questionnaires 
the participants were briefed about the purpose of the study and their task. They were also informed about the approximate time required to complete the questionnaires. After obtaining the consent of the participants, the aforementioned questionnaires were administered as per the standard instructions of each questionnaire. The questionnaire/scales were filled by the participants in the presence of either the second or the third author. All participants were requested to ensure that they have responded to each item of every questionnaire/scale.

\section{Results}

In order to examine how and to what extent the emotion regulation difficulties are associated with alexithymia, alexithymic and non-alexithymic individuals were compared on various dimensions of emotion regulation difficulties using one way ANOVA. Here it is important to mention that rather than using MANOVA, multiple univariate ANOVAs were conducted because of the exploratory nature of the study. The obtained results (including mean and SD of scores on various dimensions of emotion regulation difficulties) are presented in Table 1.

Table 1. Comparison of alexithymic and non-alexithymic participants on various dimensions of difficulties in regulating emotions

\begin{tabular}{lcccccc}
\hline & \multicolumn{2}{c}{$\begin{array}{c}\text { Non-Alexithymic } \\
\text { (N=26) }\end{array}$} & \multicolumn{3}{c}{$\begin{array}{c}\text { Alexithymic } \\
\text { (N=27) }\end{array}$} \\
\hline Dimensions of DERS & \multicolumn{2}{c}{ Mean } & SD & Mean & SD & F-ratio \\
\hline $\begin{array}{l}\text { Non-acceptance of emotional responses } \\
\text { Difficulties engaging in goal directed }\end{array}$ & 11.65 & 3.57 & 17.25 & 3.59 & $32.40^{* *}$ \\
behavior & & 4.51 & 17.07 & 4.15 & $6.66^{*}$ \\
Impulse control difficulties & 11.73 & 5.40 & 16.77 & 5.56 & $11.20^{* *}$ \\
Lack of emotional awareness & & 14.34 & 4.27 & 15.44 & 3.59 & 1.03 \\
Limited access to emotion & regulation & 15.76 & 5.42 & 20.40 & 6.03 & $29.89^{* *}$ \\
strategies & & & & & & \\
Lack of emotional clarity & & 7.00 & 1.67 & 13.37 & 3.85 & $60.61^{* *}$ \\
DERS total & 74.50 & 15.51 & 104.30 & 18.79 & $39.41^{* *}$ \\
\hline
\end{tabular}

It is evident from the table that as compared to non-alexithymics, alexithymic participants scored significantly higher on all the dimensions of DERS-H except one dimension - lack of emotional awareness. Although a statistically significant difference was not observed on this dimension of DERS-H, the observation of mean scores suggest that alexithymic individuals tend to have a greater lack of emotional awareness as compared to their non-alexithymic counterparts. One reason for the observed non-significant differences on this dimension of emotion regulation 
difficulty may be the poor reliability of this subscale of the DERS-H (alpha = .606; Pandey et al., 2010).

Overall, the findings suggest that various types of emotion regulation difficulties characterize alexithymic individuals. In other words, alexithymic and non-alexithymic individuals may be differentiated on the basis of their profile of emotion regulation difficulties. However, this analysis does not tell how accurately the alexithymic individuals may be differentiated from their non alexithymic counterparts on the basis of their emotion regulation difficulties. Thus, to address this objective, a discriminant function analysis was performed using various dimensions of emotion regulation difficulties as predictor and the alexithymic/non-alexithymic groups as categorical criterion. Since only two groups are involved, the analysis yielded only one discriminant function that was found to be statistically significant (Wilk's lambda $=0.407, \mathrm{p}<0.001)$.

The classification results (Table 2) revealed that based on the scores on various dimensions of emotion regulation difficulties, $88.9 \%$ alexithymics were accurately classified as alexithymics and only $11.1 \%$ were misclassified as non-alexithymics. However, the classification accuracy for non-alexithymics (92.3\%) was relatively higher than alexithymics and the misclassification rate was also lower $(7.7 \%)$.

Table 2. Classification results of discriminant function analysis using various dimensions of emotion regulation difficulties as predictors

\begin{tabular}{lcccc}
\hline Original group membership & \multicolumn{3}{c}{ Predicted group membership } \\
\cline { 2 - 5 } & \multicolumn{2}{c}{ Non-alexithymics } & \multicolumn{2}{c}{ Alexithymics } \\
\hline \multirow{2}{*}{ Non-alexithymics } & $\mathrm{N}$ & percentage & $\mathrm{N}$ & percentage \\
Alexithymics & 24 & 92.30 & 2 & 7.70 \\
\hline
\end{tabular}

The perusal of the discriminant function structure matrix (Table 3) revealed that three dimensions of emotion regulation difficulties, namely lack of emotional clarity, nonacceptance of emotional responses, and limited access to emotion regulation strategies, were relatively more important than others in differentiating alexithymics from non-alexithymics inasmuch as these dimensions load high on the discriminant function. The step-wise discriminant function analysis, however, revealed that only lack of emotional clarity (standardized canonical discriminant function coefficients $=.784$ ) and non-acceptance of emotional responses (standardized canonical discriminant function coefficients $=.415$ ) contributed most in differentiating alexithymic and non-alexithymics individuals with almost similar accuracy level as achieved by taking all dimensions of emotion regulation difficulties into account. 
Table 3. The discriminant function structure matrix

\begin{tabular}{lc}
\hline Dimensions of DERS-H & Function-1 \\
\hline Lack of emotional clarity & .90 \\
Non acceptance of emotional responses & .66 \\
Limited access to emotion regulation strategies & .63 \\
Impulse control difficulties & .39 \\
Difficulties engaging in goal directed behaviour & .30 \\
Lack of emotional awareness & .12 \\
\hline
\end{tabular}

The classification matrix obtained for the stepwise discriminant function analysis has been presented in Table 4 which reveals that $85.2 \%$ of alexithymic and $96.2 \%$ of nonalexithymic participants were accurately classified as alexithymic and nonalexithymic, respectively. Furthermore, the classification accuracy obtained on the basis of only the abovementioned two dimensions of the DERS-H was approximately similar to that obtained using all the dimensions of emotion regulation difficulties.

Table 4. Classification results of stepwise discriminant function analysis

\begin{tabular}{ccccc}
\hline Original group membership & \multicolumn{3}{c}{ Predicted group membership } \\
\cline { 2 - 5 } & \multicolumn{2}{c}{ Non-alexithymics } & \multicolumn{2}{c}{ Alexithymics } \\
\hline & $\mathrm{N}$ & Percentage & $\mathrm{N}$ & percentage \\
Non-alexithymics & 25 & 96.20 & 1 & 3.80 \\
Alexithymics & 4 & 14.80 & 23 & 85.20 \\
\hline
\end{tabular}

The findings of the previously mentioned analyses suggest that as compared to nonalexithymics, the alexithymics showed grater emotion regulation difficulties, specifically lack of emotional clarity and non-acceptance of emotional responses. Moreover, the various dimensions of emotion regulation difficulties differentiated between alexithymics and non-alexithymics with about $90 \%$ accuracy but two dimensions - lack of emotional clarity and non-acceptance of emotional responses contributed most to this classification accuracy. These observations imply that alexithymia is characterized by various types of emotion regulation difficulties; the lack of emotional clarity and non-acceptance of emotional responses are the core emotion regulation difficulties associated with alexithymia.

The aforesaid findings, although suggesting that alexithymia is associated with emotion regulation difficulties, do not tell whether this association is because of the overlap of these constructs or not. To address the issue a factor analysis (principal 
component analysis) was conducted on the inter-correlation matrix obtained by pooling the various dimensions of emotion regulation difficulties and alexithymia together. The principal component analysis was carried out on the whole sample of 104 participants from which the alexithymic and non-alexithymic participants were screened. Thus, the sample size was adequate for factor analysis as for each variable the number of participants was more than 10 (Kass \& Tinsley, 1979).

The principal component analysis yielded two components with eigenvalues greater that one and jointly explained $66.34 \%$ of the total variance $144.118 \%$ by the first component and $20.222 \%$ by the second). The scree plot also indicated that only two components reach significance and thus these two components were rotated using varimax rotation. The cut-off loading .60 was used for interpreting the factors as the sample size was small. The rotated component matrix is presented in Table 5.

Table 5. Rotated factor structure matrix obtained by factor analyzing the various dimensions of emotion regulation difficulties and alexithymia

\begin{tabular}{lll}
\hline & Factor 1 & Factor 2 \\
\hline Limited access to emotion regulation strategies & .88 & \\
Impulse control difficulties & .85 & \\
Non acceptance of emotional responses & .84 & \\
Difficulty in identifying feelings & .73 & \\
Lack of emotional clarity & .72 & .71 \\
Difficulty engaging in goal directed behavior & .70 & .68 \\
Externally oriented thinking & & .61 \\
Lack of emotional awareness & & \\
Difficulty in describing feelings & & \\
\hline
\end{tabular}

It is evident from Table 5 that all the dimensions of emotion regulation difficulties loaded the first factor except lack of emotional awareness that loaded the second factor. Similarly, all the dimensions of alexithymia loaded the second factor except difficulty in identifying feelings that loaded the first factor. The first factor, thus, may be labelled as emotion regulation difficulties that also include one dimension of alexithymia (difficulty in identifying feelings). Similarly, the second factor may be labelled as alexithymia. However, this factor also included lack of emotional awareness that has been originally measured as a dimension of emotion regulation difficulties. This pattern of cross loading of one dimension of each construct on both the factors suggests only minimal overlap between the constructs inasmuch as most of the other dimensions of each construct loaded separate factors. The findings, thus, imply that emotion regulation difficulties and alexithymia are largely independent constructs with minimal content overlap. 
To test the hypothesis that the poor mental health of alexithymic individuals may be caused by their difficulties in regulating emotions, the alexithymic and nonalexithymic individuals were compared on various dimensions of mental health with and without controlling the effect of various types of emotion regulation difficulties. The data was analyzed using one way analysis of co-variance (ANCOVA) design with mental health as dependent measure and various dimensions of emotion regulation difficulties as covariate. In one analysis all the dimensions of emotion regulation difficulties were entered as covariates while in the other only those two dimensions were used as covariates that emerged as best predictor for differentiating alexithymics from non-alexithymics. However, before controlling the covariance of emotion regulation difficulties one way ANOVA was conducted using various dimensions of mental health as dependent measure to ensure that alexithymic individuals reported significantly more mental health related problems as compared to non-alexithymics. The obtained results are presented in Table 6.

Table 6. The comparison of mental health of alexithymics and non-alexithymics before and after controlling the variance attributable to emotion regulation difficulties

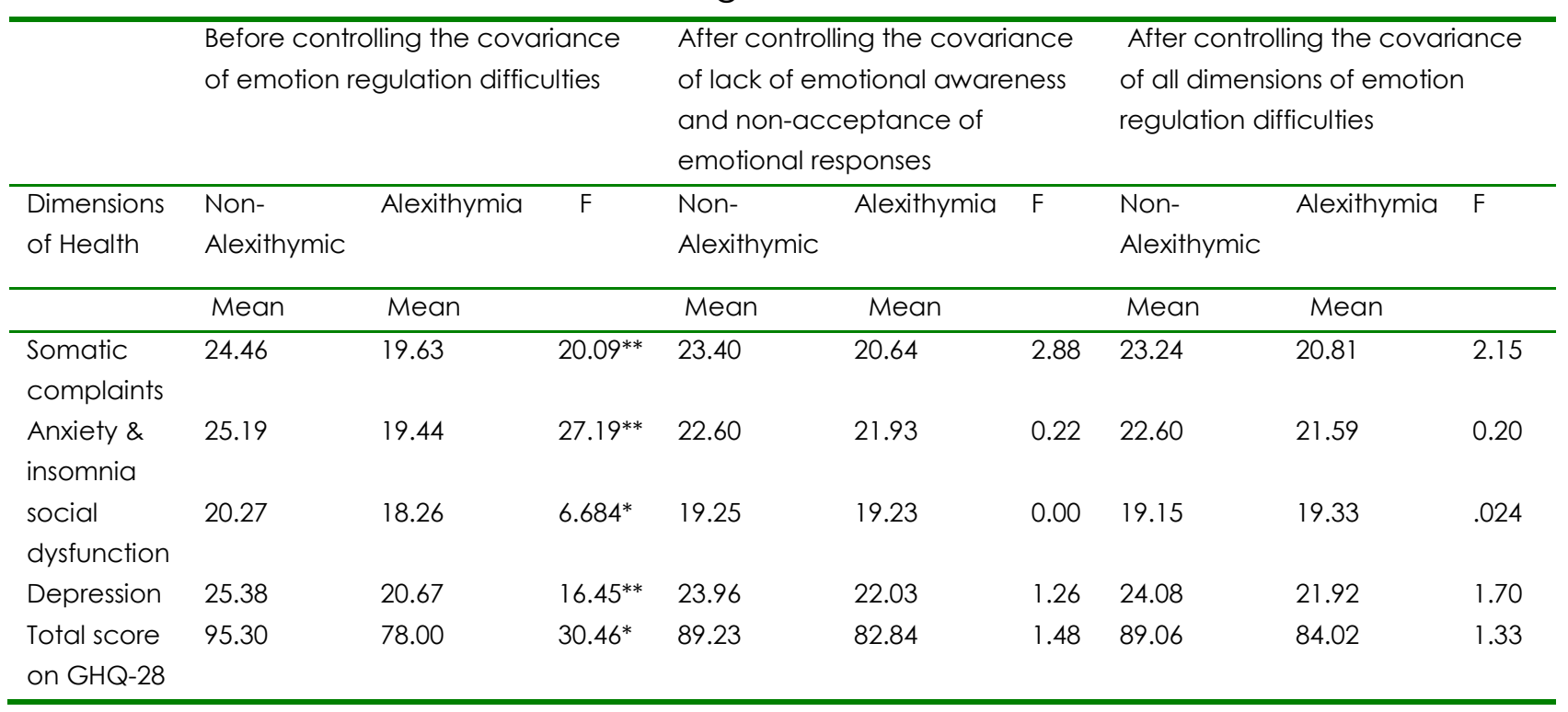

The findings revealed that there were significant differences between alexithymic and non-alexithymic individuals on all dimensions of mental health before controlling for the contribution of difficulties in emotion regulation. The alexithymic individuals scored significantly lower than non-alexithymics on measures of mental health. Since low score on various dimensions of the GHQ-28 indicate greater severity/frequency of symptoms, the observed pattern of mean difference implies that alexithymic individuals (as compared to non-alexithymics) reported more somatic complaints, depressive symptoms and symptoms of anxiety, insomnia, and social dysfunction. However, after controlling for the contribution of emotion regulation difficulties, the 
difference between alexithymic and non-alexithymic individuals became statistically non-significant on all the dimension of mental health. Furthermore, the difference in mental health between alexithymic and non-alexithymic disappeared not only after controlling the effect of all the dimensions of emotion regulation difficulties but also after controlling the effect of only those two dimensions of emotion regulation difficulties that were found as being the best discriminator of alexithymics and nonalexithymics. The findings, thus, imply that emotion regulation difficulties, especially the lack of emotional clarity and non-acceptance of emotional responses, mediate the effect of alexithymia on certain dimensions of mental health, namely depression, anxiety and insomnia, somatic complaints, and social dysfunction. In other words, the findings imply that the poor mental health observed in alexithymic individuals may be largely due to their difficulties in regulating emotions.

\section{Discussion}

The findings of the present study suggest that alexithymia is associated with greater emotion regulation difficulties and a minimum overlap between these constructs was observed. These findings propose that alexithymia is an independent construct and its relationship with emotion regulation may reflect the interdependent nature of these constructs. Further, the findings of the present study add to the existing literature by demonstrating that the elevated mental health problems in alexithymic individuals may be because of their emotion regulation difficulties as indicated by a non-significant difference between alexithymics and non-alexithymics in terms of mental health after statistically controlling the effect of emotion regulation difficulties.

The earlier theoretical proposition that alexithymia may be considered as a disorder of emotion regulation (Taylor, 1994; Taylor, Bagby, \& Parker, 1997) is empirically supported by the present observation that alexithymics showed a variety of emotion regulation difficulties such as non-acceptance of emotional responses, lack of emotional clarity, difficulties engaging in goal-directed behaviour, impulse control difficulties, and limited access to emotion regulation strategies. The observation that these emotion regulation difficulties can differentiate between alexithymic individuals and non-alexithymics with around $90 \%$ accuracy and that lack of emotional clarity and non-acceptance of emotional responses (the two specific components of emotion regulation difficulties) contribute most to the observed discrimination accuracy provides further support to the above speculation. The finding that certain specific emotion regulation difficulties such as lack of emotional clarity and non-acceptance of emotional responses are relatively more prominent in 
alexithymic individuals also expands our understanding of the nature of emotional deregulation in alexithymia.

The finding that alexithymia is associated with (or characterized by) emotion regulation difficulties suggests several possibilities about the nature of the relationship of these constructs. This relationship may indicate that the two constructs overlap with each other and thus have very similar content or behavioural domain. Apart from overlapping relationship (i.e. similarity of defining behavioural domain/content), the observed association of alexithymia and emotion regulation difficulties may reflect interdependence of the construct. In other words, the one construct may be dependent on or caused by the other construct and its subdomains. For instance, the interdependence hypothesis may suggest that alexithymia leads to emotion regulation difficulties.

The reported theoretical coherence between the constructs of alexithymia and emotion regulation difficulties lends support to the overlap hypothesis but the findings of the factor analysis that all the dimensions of emotion regulation difficulties and that of alexithymia loaded separate factors (with one exception) questions the sustenance of this hypothesis. One dimension of emotion regulation difficulties (lack of emotional awareness) loaded other dimensions of alexithymia and one dimension of alexithymia (difficulty in identifying feelings) loaded other dimensions of emotion regulation difficulties. The review of item content of these dimensions reveals substantial similarity and thus may be considered to reflect the same underlying deficit in understanding of emotions. This similarity of content and underlying process of difficulty in identifying feelings (a dimension of alexithymia) and lack of emotional awareness (a dimension of emotion regulation difficulties) may be a probable reason behind the interchange (cross-loading) of these dimensions in the factor analysis. Taking these dimensions as substitute of each other the orthogonality and factorial purity of the observed two factors (factor 1: emotion regulation difficulties; factor 2: alexithymia) seems less questionable. Thus, alexithymia and emotion regulation difficulties may be considered orthogonal (non-overlapping) constructs. However, the similarity of content of the abovementioned dimensions of the two constructs does suggest that difficulty in emotional understanding (reflected by either the difficulty in identifying feelings or lack of emotional awareness) forms a common substance for both the constructs and may be a region of overlap. To sum up, the findings of the factor analysis do not completely rule out the possibility of overlap between alexithymia and emotion regulation difficulties but definitely suggest that both constructs are largely independent and there is only a minimum overlap between them. 
Based on our own findings and the earlier observations we can speculate that the observed association between alexithymia and emotion regulating difficulties may be more an indicator of interdependence rather than content overlap. More specifically, we assume that the type of cognitive/emotional deficits associated with alexithymia may lead to various types of emotion regulation difficulties. The alexithymics' difficulty in cognitive modulation of emotional responses (Pandey, 1995), difficulty in identifying and describing emotions (Taylor, 1994; Taylor, 1984) coupled with poor emotional differentiation ability, may contribute to their poor emotion regulation ability (Lane \& Schwartz, 1987). It has been noted that individuals with poorly differentiated emotional experiences were found to be less able to regulate emotion as compared to those who have highly differentiated emotional experiences (Barrett, Gross, Christensen, \& Benvenuto, 2001) and that adaptive emotion regulation involves awareness and understanding of emotions and the capacity for monitoring and evaluating emotional experiences (Lane \& Schwartz, 1987). It is well documented that alexithymic individuals are lacking when it comes to understanding and awareness of emotions (Taylor, 1994; Taylor, 1984) and thus, the observed emotional regulation difficulties in alexithymic individuals appears to be theoretically congruent with a conceptualization of emotion regulation difficulties in terms of lack of emotional awareness and differentiation (Gratz \& Roemer, 2004).

Another signficant contribution of the current study is that it provides some preliminary evidence to the theoretical speculation that emotion regulation difficulties associated with alexithymia may be a factor that enhances the risk of developing mental health problems among alexithymic individuals. The findings of the present study revealed that, when compared to non-alexithymics, alexithymic individuals reported significantly greater symptoms of depression, anxiety, insomnia, somatic complaints and showed more social dysfunction. However, this difference in the self-reported mental health status of alexithymics and non-alexithymics became statistically non-significant when the contribution of emotion regulation difficulties in general and lack of emotional clarity and non-acceptance of emotional responses in particular were statistically controlled for. This observation supports the speculation that emotion regulation difficulty acts as a mediating factor in the observed relationship between alexithymia and mental health problems. Despite the partial overlap between alexithymia and emotion regulation difficulties, the findings of the present study do indicate the possibility that the a variety of other emotion regulation difficulties associated with alexithymia (particularly the nonoverlapping components of emotion regulation difficulties) may potentially contribute to elevated mental health problems (such as depression, anxiety, somatic complaints etc.) among alexithymic individuals. A number of earlier observations and theoretical propositions suggest that emotion regulation difficulties are at the 
core of mental health problems (Gross \& Munoz, 1995). In a recent meta analytic review, Aldao and colleagues (Aldao, Nolen-Hoeksema, \& Schweizer, 2010) have noted that various types of psychopathology and mental health problems can be viewed as an outcome of emotion regulation difficulties. They further reported that mental disorders like anxiety and depression were more consistently associated with emotion regulation difficulties. Similarly, some researchers have theorized that difficulties in emotion regulation may also lead to poor interpersonal relationship (Gross \& Munoz, 1995) which in turn may result in social dysfunction and other mental health problems.

Going over the major observations of the present study it can be concluded that alexithymia is characterized by a variety of emotion regulation difficulties and these emotional difficulties associated with alexithymia contribute to mental health related problems. Furthermore, the findings also suggest that, except for the deficit in emotional understanding, the constructs of alexithymia and emotion regulation difficulties represent a different set of affective difficulties and should be considered orthogonal constructs with minimal overlap. However, the observed overlap between one dimension of each construct suggests the possibility that emotion regulation difficulty may be a broader construct representing a spectrum of emotion related difficulties and alexithymia (or its some component like difficulty in identifying emotions) may be a specific point on this spectrum. Such speculation, however, needs further empirical verifications before drawing any conclusion inasmuch as the findings of our factor analysis requires further cross-validation.

Though the findings of the study are encouraging and have important implications for practicing clinical and health psychologists, it would be premature to make any recommendations and generalization because the findings are based on a small sample taken from a very specific population of students. As the health related issues and problems of students and young adults as well as their way and capacity to handle emotions may be very different from that of other age groups and population, it would be difficult to generalize these findings to other populations. Moreover, the findings of the present study based on factor (principal component) analysis also need to be replicated on a relatively large sample before their generalization inasmuch as the factor structure obtained on a small sample (in the present case $\mathrm{N}=104$ ) is less likely to be stable. 


\section{References}

Aldao, A., Nolen-Hoeksema, S., \& Schweizer, S. (2010). Emotion-regulation strategies across psychopathology: A meta-analytic review. Clinical Psychology Review, 30 (2), 217237. doi:10.1016/j.cpr.2009.11.004

Bamonti, P. M., Heisel, M. J., Topciu, R. A., Franus, N., Talbot, N. L., \& Duberstein, P. R. (2010). Association of alexithymia and depression symptom severity in adults aged 50 years and older. American Journal of Geriatric Psychology, 18(1), 51- 56.

Barrett, F., Gross, J. J., Christensen, C. T., \& Benvenuto, M. (2001). Knowing what you're feeling and knowing what to do about it: Mapping the relation between emotion differentiation and emotion regulation. Cognition and Emotion, 15(6), 713-724.

Bavermann, T. M., Parker, J. D. A., \& Taylor, G.J. (2002). Alexithymia and sleep disorder symptoms. Sleep, 25, A389-A389.

Bavermann, T. M., Parker, J. D. A., \& Taylor, G. J. (2008). Sleep problems and sleep hygiene in young adults with alexithymia. Personality and Individual Differences, 45(4), 318-322. doi:10.1016/j.paid.2008.04.019

Bekendam, C.C. (1997). Dimensions of emotional intelligence: Attachment, affect regulation, alexithymia and empathy. Dissertation Abstracts International, 58(4-B), $2109-$ 2116.

Byrne, N., \& Ditto, B. (2005). Alexithymia, cardiovascular reactivity, and symptom reporting during blood donation. Psychosomatic Medicine, 67, 471-475.

Campbell-Sills, L., Barlow, D. H., Brown, T. A., \& Hofmann, S. G. (2006). Acceptability and suppression of negative emotion in anxiety and mood disorders. Emotion, 6(4), 587-595.

Cole, P. M., Michel, M. K., \& Teti, L. O. (1994). The development of emotion regulation and dysregulation: A clinical perspective. Monographs of the Society for Research in Child Development, 59(2/3), 73-100.

Connelly, M., \& Denney, D. R. (2007). Regulation of emotions during experimental stress in alexithymia. Journal of Psychosomatic Research, 62 (6), 649-656.

Devine, H., Stewart, S. H., \& Watt, M. C. (1999). Relations between anxiety sensitivity and dimensions of alexithymia in a young adult sample. Journal of Psychosomatic Research, $47(2), 145-158$. 
Garner, P. W., \& Spears, F. M. (2001). Emotion regulation in low-income preschoolers. Social Development, 9(2), 246-264.

Goldberg, D. P., \& Hillier, V. F. (1979). A scaled version of the General Health Questionnaire. Psychological Medicine, 9(01), 139-145.

Gratz, K. L., \& Roemer, L. (2004). Multidimensional assessment of emotion regulation and dysregulation: Development, factor structure, and initial validation of the difficulties in emotion regulation scale. Journal of Psychopathology and Behavioral Assessment, 26 (1), $41-54$.

Gratz, K. L., Tull, M. T., \& Gunderson, J. (2008). Preliminary data on the relationship between anxiety sensitivity and borderline personality disorder: The role of experiential avoidance. Journal of Psychiatric Research, 42 (7), 550-559.

Greenberg, L. S., \& Paivio, S. C. (1998). Allowing and accepting painful emotional experiences. International Journal of Action Methods, 51, 47-62.

Gross, J. J., \& Munoz, R. F. (1995). Emotion regulation and mental health. Clinical Psychology: Science and Practice, 2(2), 151-164.

Hayes, S. C., Strosahl, K. D., \& Wilson, K. G. (1999). The ACT model of psychopathology and human suffering. In Acceptance and commitment therapy: An experiential approach to behavior change (p. 49-80). New York: Guilford Press.

Honkalampi, K., Koivumaa-Honkanen, H., Lehto, S., Hintikka, J., Haatainen, K., Rissanen, T., \& Viinamaki, H. (2010). Is alexithymia a risk factor for major depression, personality disorder, or alcohol use disorders? A prospective population-based study. Journal of Psychosomatic Research, 68(3), 269-273. doi:10.1016/j.jpsychores.2009.05.010

Jula, A., Salminen, J. K., \& Saarijarvi, S. (1999). Alexithymia: A facet of essential hypertension. Hypertension, 33, 1057-1061.

Kass, R. A., \& Tinsley, H. E.A. (1979). Factor analysis. Journal of Leisure Research, 11, 120138

Kessler, H., Kammerer, M., Hoffmann, H., \& Trave, H. C. (2010). Regulation of emotions and alexithymia: A correlative study. Psychotherapie, Psychosomatik, Medizinische Psychologie, 60(5), 169-174. doi:10.1055/s-0029-1234046 
Lane, R. D., \& Schwartz, G. E. (1987). Levels of emotional awareness: A cognitivedevelopmental theory and its application to psychopathology. The American Journal of Psychiatry, 144 (2), 133.

McDermott, M., Tull, M., Gratz, K., Daughters, S., \& Lejuez, C. (2009). The role of anxiety sensitivity and difficulties in emotion regulation in posttraumatic stress disorder among crack/cocaine dependent patients in residential substance abuse treatment. Journal of Anxiety Disorders, 23(5), 591-599. doi:10.1016/j.janxdis.2009.01.006

Mennin, D. S., McLaughlin, K. A., \& Flanagan, T. J. (2009). Emotion regulation deficits in generalized anxiety disorder, social anxiety disorder, and their co-occurrence. Journal of Anxiety Disorders, 23(7), 866-871.

Pandey, R. (1995). Stroop interference effect of emotion-arousing words in alexithymia. Journal of the Indian Academy of Applied Psychology, 21, 21-28.

Pandey, R., Mandal, M. K., Taylor, G. J., \& Parker, J. D. A. (1996). Cross-cultural alexithymia: Development and validation of a Hindi translation of the 20-item Toronto Alexithymia Scale. Journal of Clinical Psychology, 52 (2), 173-176.

Pandey, R., Saxena, P., Gupta, G., \& Dubey, A. (2010). Development and psychometric evaluation of a Hindi version of the Difficulties in Emotion Regulation Scale. Unpublished Manuscript, Department of Psychology, Banaras Hindu University, Varanasi, India.

Parker, J. D. A., Taylor, G. J., \& Bagby, R. M. (1998). Alexithymia: Relationship with ego defence and coping styles. Comprehensive Psychiatry, 39(2), 91-98.

Parker, J. D. A., Taylor, G. J., Bagby, R. M., \& Acklin, M. W. (1993). Alexithymia in panic disorder and simple phobia- A comparative study. American Journal of Psychiatry, 150(7), 1105-1107.

Schaffer, C. E. (1993). The role of adult attachment in the experience and regulation of affect. Mikrofiche-Ausg.: Ann Arbor, Mich.: Univ. Microfilms Intern.

Scheidt, C. E., \& Waller, E. (1999). Attachment representation, affect regulation and psychophysiological reactivity : Comments on the relevance of current findings in the field of attachment research for psychosomatic medicine. Zeitschrift fur Psychosomatische Medizin und Psychotherapie, 45 (4), 313-332.

Taylor, G. J. (1984). Alexithymia: Concept, measurement, and implications for treatment. American Journal of Psychiatry, 141 (6), 725-732. 
Taylor, G. J. (1994). The alexithymia construct: Conceptualization, validation, and relationship with basic dimensions of personality. New Trends in Experimental \& Clinical Psychiatry, 10, 61-74.

Taylor, G. J., Bagby, R. M., \& Parker, J. D. A. (1997). Disorders of affect regulation. Cambridge,UK : Cambridge University Press.

Tull, M. T., \& Roemer, L. (2007). Emotion regulation difficulties associated with the experience of uncued panic attacks: Evidence of experiential avoidance, emotional nonacceptance, and decreased emotional clarity. Behavior Therapy, 38 (4), 378-391.

Whiteside, U., Chen, E., Neighbors, C., Hunter, D., Lo, T., \& Larimer, M. (2007).Difficulties regulating emotions: Do binge eaters have fewer strategies to modulate and tolerate negative affect? Eating Behaviors, 8(2), 162-169.

About the authors:

Dr. Rakesh Pandey is presently professor at the Department of Psychology, Banaras Hindu University, India. His current research is focused on exploring the link of emotion and health and includes such topics as alexithymia, emotional intelligence, emotion regulation, and emotion related difficulties and deficits in health and well-being. He is also working on an ongoing UGC funded project on hemispheric asymmetry in depression.

Address for correspondence: Rakesh Pandey, Professor, Department of Psychology, Banaras Hindu University, Varanasi-221005, India

Email:rpan_in@yahoo.com rpandeybhu@gmail.com

Prachi Saxena is currently perusing her Ph.D. research in department of Psychology, Banaras Hindu University. Her work is centered on role of emotional deficits in substance abuse - mental illness relationship.

E-mail: prachisaxena.bhu@gmail.com

Akanksha Dubey is a Ph.D. student in the department of Psychology, Banaras Hindu University. Currently, she is perusing her Ph.D. on the role of emotion processing in mental health.

E-mail: akanksha.dubey31@gmail.com 